le type d'université dit "progressiste", et cela, sans aucun souci d'objectivité, bien entendu. Je me sentirais à l'aise dans ce type d'université où la présence d'un syndicalisme utilitaire et revendicatif au plan économique et professionnel laisserait une large place à l'autogouvernement, du moins au niveau des unités administratives et pédagogiques de base. Je me sentirais d'autant plus à l'aise que j'épouse les quelques valeurs fondamentales d'une société libérale et "démocratique" et que je privilégie, à mon propre insu, une vision cohérente, harmonieuse des systèmes sociaux, tout en reconnaissant les fonctions positives et innovatrices des conflits inhérents au fonctionnement de tout système social. En utilisant un modèle analy tique qui s'inspire beaucoup plus de l'approche structuro-fonctionnelle, les auteurs, à leur propre insu, évitent difficilement cette tendance à privilégier une vision cohérente des systèmes sociaux où la notion d'équilibre dynamique occupe une place importante. Cette réflexion rejoint celle de monsieur Raymond Laliberté qui est beaucoup plus poussée et qu'on retrouve dans la deuxième partie de l'ouvrage avec les réflexions des autres membres du panel que les auteurs ont consultés.

Somme toute, les auteurs nous fournissent une multitude de typologies et beaucoup d'éléments de réflexion qui pourraient servir à concevoir et mettre en place un projet de recherche empirique dont l'objet serait d'évaluer l'impact réel d'un type ou l'autre de syndicalisme sur la structure et le fonctionnement de l'université.

Laurent Bélanger

Université Laval

"La négociation collective dans le milieu universitaire," Relations Industrielles, Vol. 30, no. 4, 1975, pp. 643-736.

La revue Relations Industrielles publie dans ce numéro des communications présentées au Congrès annuel de l'Institut canadien de recherche en relations industrielles, tenu à Edmonton en Juin 1975. Comme il s'agit dans plusieurs cas d'expressions d'opinions personnelles, dans une large mesure, il est préférable de commenter les articles un à un.

H. D. Woods, "Collective Bargaining and Academic Freedom. Are they compatible?", pp. 643-62.

Le professeur Woods estime que les universités sont, peut-être, les institutions les plus démocratiques en Occident (p. 645). Les associations de professeurs seraient largement responsables de cette situation et la transformation de ces associations volontaires en syndicats accrédités risque de mettre en péril la liberté académique en mettant l'accent sur la division entre l'autorité hiérarchique et le corps professoral.

Qu'il y ait eu une forte décentralisation du pouvoir dans les années 1950 et 1960, nul ne saurait le nier. Peut-être aussi les associations de professeurs ont-elles été un facteur puissant dans cette évolution. Mais je ne crois pas que les universités soient des modèles de démocratie. Il s'en faut de beaucoup. En particulier, j'ai l'impression que le paternalisme y est encore présent, peut-être plus qu'avant, et c'est la syndicalisation seule qui transformera certains privilèges que nous avons en des droits dûment reconnus par une convention collective. 
Notons au passage que certains privilèges discutables, sinon indus, peuvent être perdus à l'occasion de ces changements.

Relevons deux autres points parmi plusieurs.

D’abord, la liberté dont nous jouissons dans la détermination du contenu de nos cours m'apparaît plutôt négative. Ce n'est pas tellement un droit qu'une situation de fait qui résulte de l'incapacité de contrôler directement ce contenu, de la part de l'autorité hiérarchique comme d'ailleurs des étudiants, en raison du caractère hautement spécialisé de nos enseignements. Quant aux contrôles indirects, il sont connus. Mentionnons pour mémoire les mécanismes de sélection, de nomination et de promotion des professeurs.

Ceci m'amène au deuxième point. Selon mon expérience, l'assemblée départementale n'a pas le contrôle en matière d'engagement et de promotion des professeurs même si l'initiative en revient au département. Bien sûr, lorsque le dossier respecte toutes et chacune des normes officielles, les étapes sont franchies sans accrochage (quoique trop lentement, à mon avis). Dans le cas contraire, la balance du pouvoir, est entre les mains des "autorités" et le fait qu'elles soient largement consitutées d'anciens professeurs ne me paraît pas être un facteur toujours positif. Mais sur ce point, je n'ai pu à ce jour me faire une opinion ferme.

Donald D. Carter, "Collective Bargaining in Canadian Colleges an Universities. Some unresolved dilemmas", pp. 662-74.

L'auteur note les réticences des professeurs d'université à adopter le modèle standard de négociation collective. Cette hésitation provient d'un dilemme fondamental: Vaut-il mieux risquer de perdre leur statut social et institutionnel en essayant d'améliorer par l'action collective leur statut économique ou l'inverse est-il préférable?

Ce dilemme revêt plusieurs formes. En premier lieu, l'action collective entre en contradiction avec certaines valeurs "professionnalistes" et individualistes des professeurs. Deuxièmement, elle peut nuire à la participation à la gestion des institutions. Troisièmement, la négociation collective implique un choix entre la sécurité d'emploi et le niveau de rémunération, compte tenu des restrictions budgétaires imposées aux universités par les gouvernements dans les années récentes. Enfin l'action collective entraînera nécessairement l'entrée en lice des gouvernements, ce qui veut dire un contrôle accru et la possibilité d'une impasse.

Ce qui frappe dans son analyse c'est la notion d'action collective, pouvant revêtir plusieurs formes y compris l'association volontaire et l'association accréditée, et qui est opposée à l'inaction pure et simple. C'est-à-dire la détermination unilatérale des conditions de travail par l'administration universitaire et les gouvernements. Cela a le mérite de tenir compte de l'éventail complet des situations réelles.

Par contre, l'auteur a sous-estimé le potentiel de militantisme des professeurs, du moins comme la situation au Québec l'a montré par la suite. Cela d'ailleurs tend à confirmer l'hypothèse selon laquelle les universités francophones et anglophones ont une attitude différente face à la négociation collective.

Jean Boivin, "La négociation collective chez les professeurs d'université. Quelques cas", pp. 674-707.

Cet exposé est le plus long de tous et défie toute tentative de résumé détaillé. Il se divise en deux parties. 
Dans une première, l'auteur se demande si le phénomène de syndicalisation amorcé aux Etats-Unis, au Canada et au Québec dans les années soixante va se généraliser ou se résorber (pp. 674-5). L'étude n'apporte pas la réponse à cette question, probablement parce que, à mon sens, cette réponse n'existe pas, ne serait-ce qu'en raison de la diversité même des situations où se trouvent les universités et leurs professeurs e.g. aux U.S.A. les universités de type Ivy League et privées par comparaison aux universités publiques offrant des diplômes de premier cycle seulement, et au Québec, la différence d'attitude vis-à-vis la syndicalisation sur les campus francophones et anglophones. (Ces deux exemples sont tirés de l'étude de Jean Boivin).

D'autres part, cette première partie contient une série d'informations considérable, allant du pourcentage de pénétration syndicale dans les universités américaines jusqu'à une revue sommaire de la détermination des unités d'accréditation. Le tout est cependant quelque peu disparate et certains détails sont gratuits.

Dans la seconde partie le professeur Boivin examine quelques conventions collectives afin de découvrir les principaux problèmes auxquels les professeurs syndiqués se sont attaqués (j'ajouterais: avec succès) dans leurs négociations (p. 688). Si l'on s'en tient aux universités québécoises (UQUAM et UQUAR), il apparaît que les syndicats de professeurs ont pu à la fois gagner des avantages semblables à ceux des autres travailleurs syndiqués (e.g. l'arbitrage en matière du congédiement) et négocier des clauses plus inusitées (e.g. des dispositions traitant de la participation des syndiqués à la gestion). Si on se rapporte à la situation qui prévalait dans les universités avant la syndicalisation, ces deux exemples nous amèneraient à conclure que c'est sur le premier terrain que les gains les plus nets ont été enregistrés.

Sur la fois de l'exposé de Jean Boivin, je concluerais également que des résultats obtenus au Québec par les syndicats de professeurs sont nettement plus concluants que ce que nos confrères américains ont pu réaliser.

David M. Beatty, "Collective Bargaining in the University Faculties. Pros and Cons", pp. 202-27.

Le professeur Beatty croit que le syndicalisme universitaire ne va pas changer les objectifs fondamentaux des universités. Bien au contraire la syndicalisation serait plutôt une réaction contre des forces extérieures affectant ces objectifs e.g. le rôle accru des gouvernements.

De plus, le syndicalisme n'est pour lui qu'un modèle de processus de décision et il est relativement neutre face aux objectifs de l'institution universitaire. Les demandes syndicales dépendront en conséquence des besoins tels que perçus par les membres et les résultats obtenus seront fonction de circonstances extérieures, telles les conditions du marché en ce qui concerne la rémunération.

Le résultat le plus dramatique de l'adoption du système de négociation collective, mise à part l'amélioration des salaires et de la sécurité d'emploi, sera de cristalliser l'opposition entre professeurs et administrateurs, au détriment de la collégialité. Ceci bénéficiera aux deux parties. Les administrateurs seront amenés à être plus efficaces; les professeurs de leur côté prendront mieux conscience de leurs besoins propres. Ainsi prendra fin l'illusion qu'ont les professeurs de participer réellement à la gestion de l'université dans le régime actuel.

La négociation collective, si elle était couronnée de succès (économique), aurait aussi pour effet de mettre en évidence deux ordres de conflits d'intérêt. D'abord le postulat de 
l'homogénéité de la communauté universitaire volerait en éclats au moment où d'autres groupes auraient à payer le prix des avantages accrus consentis aux professeurs e.g. à payer des frais de scolarité plus élevés ou des taxes plus lourdes.

D'autre part, le processus fera ressortir les conflits entre différents groupes, par exemple entre les professeurs permanents et non-permanents, les facultés professionnelles et non professionnelles. Mais, note l'auteur avec pertinence, l'ensemble du corps professoral doit savoir reconnaître que la force de négociation de l'ensemble repose sur celle des groupes les plus forts. Si tel est le cas, les divergences d'intérêt se résorberont d'elles-mêmes.

Gilbert Levine, "Collective Bargaining in University Faculties Pros and Cons", pp. 727-35.

Dans son court texte, Gilbert Levine lance un cri du coeur: La syndicalisation devrait amener les professeurs d'université à s'unir aux travailleurs "ordinaires". Si ce résultat était atteint, un bénéfice social (ou externe) s'ajouterait aux avantages directs que leur apporterait la négociation collective.

Cependant, bien que je partage ce point de vue, je douterais fort que le pont soit jeté entre les syndicats d'enseignants universitaires et le mouvement syndical traditionnel. En effet, si l'on considère que la CEQ est elle même une centrale largement dominée par une catégorie professionnelle, il ne reste que le syndicat des professeurs de l'UQUAM qui soit affilié à la CSN. Et l'UQUAM à de nombreux égards, constitue un cas particulier.

\section{EN GUISE DE CONCLUSION}

Après lecture de ces cinq essais, il serait difficile d'en déterminer le commun dénominateur. Chacun doit plutôt être considéré comme explorant une facette d'une question complexe. Dans l'ensemble, ils constituent toutefois un excellent point de départ et une source abondante de réflexion pour qui voudrait examiner sérieusement pour la première fois les implications de la syndicalisation universitaire.

Une mise en garde toutefois: compte tenu des événements récents sur le front universitaire, je pense ici au Québec surtout, il me semble que ces textes vieilliront rapidement.

Claude D'Aoust

Université de Montréal

Garnet McDiarmid, ed., From Quantitative to Qualitative Change in Ontario Education, A Festschrift for R. W. B. Jackson. Toronto: The Ontario Institute for Studies in Education, Toronto, 1976. $190 \mathrm{pp}$.

In his several roles as researcher, statistician, cabinet advisor and, for the decade 1965-75, Director of the Ontario Institute for Studies in Education, R.W.B. Jackson stimulated and nurtured the expansion and improvement of education in Ontario. In the main he worked quietly behind the seats of power, but his influential address, "The Problem of Numbers in University Enrolment," to the Canadian Education Association in 1963 was, as John Holland points out, "a nearly irrefutable brief for a mobilization of political and financial resources 